\title{
From Burnout to Well-Being: A Focus on Resilience
}

\author{
Najjia N. Mahmoud, MD, FACS, FASCRS ${ }^{1}$ David Rothenberger, MD, FACS, FASCRS² \\ ${ }^{1}$ Division of Colon and Rectal Surgery, Department of Surgery, University of \\ Pennsylvania Health System, Philadelphia, Pennsylvania \\ 2 Department of Surgery, University of Minnesota, Minneapolis, \\ Minnesota \\ Address for correspondence Najjia N. Mahmoud, MD, FACS, FASCRS, \\ Division of Colon and Rectal Surgery, Department of Surgery, 4 \\ Silverstein, University of Pennsylvania Health System, 3400 Spruce \\ Street, 4 Silverstein, Philadelphia, PA 19104 \\ (e-mail: Najjia.mahmoud@uphs.upenn.edu).
}

Clin Colon Rectal Surg 2019;32:415-423.

\begin{abstract}
Keywords

- burnout

- resilience

- engagement

- stress management

- well-being

Burnout is a widespread problem in health care. Factors that contribute to enhancing engagement and building resiliency are widely discussed, but the data supporting these practices are not well understood. Interventions aimed at increasing engagement and promoting resiliency are targeted toward individual practitioners, health care institutions, and national organizations. Knowledge of the data supporting various kinds of interventions is vital to implementing change meaningfully. Prevention of burnout should start early in training with appropriate modeling and input from mentors and should incorporate stress management strategies. The most compelling data for building resilience requires institutions, physicians, and their support staff to align their values to create a mutual culture of wellness and engagement. It is imperative that institutional and national reform allows us as physicians to preserve our relationships with patients and colleagues, while also prioritizing time to reflect and pursue outside interests that recharge and restore.
\end{abstract}

The problem of surgeon burnout and depression cannot be overstated and is discussed and evaluated in the prior chapter in detail. There exists compelling evidence that the rate of depression and burnout is increasing in the field of general surgery. This phenomenon leads to medical errors and loss of productivity and affects both younger surgeons and females to a greater degree. Critical examination of factors affecting resilience and well-being remains limited, and the data that exist are not well understood by either physicians or administrators.

It is evident that key components of burnout-emotional exhaustion, depersonalization, and depression-exist in nursing and the allied professions as well. In fact, important interventions and observations from allied professionals were instrumental in developing metrics to evaluate burnout in physicians. Interventions to promote surgeon well-being and resilience are mostly generalized from those developed in other specialties and subspecialties. Although this may be quite appropriate, as it appears that, regardless of specialty, the same stressors that affect other physicians and health care professionals also affect surgeons in much the same way, there are subtle and not-so-subtle differences in how others perceive surgeons and the work we do as well as our resultant errors. Charles Bosk famously says in Forgive and Remember: Managing Medical Failure, "When the patient of an internist dies, the natural question is ... 'What happened?' When the patient of a surgeon dies, his colleagues ask, 'What did you do?"'1

This study seeks to better describe strategies for promoting physician well-being and resilience and present them not as the "opposite" but as an "antidote" or counterpoint for burnout and depression driven by clinical activity. Furthermore, it seeks to emphasize and describe the concept of prevention. Factors affecting individual agency and those involving institutional and national prerogatives and programs to prevent burnout and promote well-being are described and evaluated. It seems very logical that emotional, intellectual, and situational factors affecting depression and burnout exist for even the most resilient surgeons. Understanding data supporting strategies to promote and maintain well-being is effective for everyone practicing surgery today.
Issue Theme Surgeon Health; Guest Editor: Jennifer S. Davids, MD, FACS, FASCRS
Copyright (C) 2019 by Thieme Medical Publishers, Inc., 333 Seventh Avenue, New York, NY 10001, USA. Tel: +1(212) 584-4662.
DOI https://doi.org/ 10.1055/s-0039-1692710. ISSN 1531-0043. 


\section{Burnout and Resilience: Why It Matters}

Physician burnout is an epidemic in the United States today. Recent recognition of the phenomenon has rapidly evolved into identification of causes, recommendations for prevention, and promotion of well-being, as well as an examination of the effects of depression and burnout on individuals, institutions, and the integrity of the U.S. health care system. The effects of burnout are wide-ranging, affecting patients, health systems, and individuals. It has been estimated that more than $60 \%$ of surgeons would retire if they had the chance, citing reasons that include increased administrative and clerical tasks, less time for collaboration, profit-driven decision-making, and increasing regulatory requirements with no clear link to improved patient outcomes. Furthermore, it has been shown that even small increases in burnout scores are associated with self-reported perceptions of major medical errors, decreased efficacy and time in practice, and suicidal ideation. ${ }^{2}$ Interventions that make small differences may have a large impact.

While most who leave practice do so at their own discretion, a staggering number of physicians are lost because of the sustained effects of burnout. Tragically, 400 physicians commit suicide every year-a statistic that amounts to the number of graduates of two medical classes every year, or over a "doctor a day." Physicians, in general, have a lower rate of mortality from other illnesses such as heart and lung disease and cancer than the general population but are near the top of the list of professions for suicide. Among medical students, suicide is the second leading cause of death behind accidents. ${ }^{3}$ Depression is the leading risk factor for suicide and is present in a higher proportion of physicians than the general population, affecting $12 \%$ of men and almost $20 \%$ of women. ${ }^{4}$ This is concordant with the fact that burnout shows a gender predilection for females. Burnout causes an insidious loss of productivity that results from early retirement, time spent doing clerical work, and high turnover. It has been estimated that the loss of yearly productivity secondary to burnout when evaluated longitudinally is equivalent to the loss of the services of seven medical school graduating classes. This represents an astonishing number of patientcare hours and an obvious huge loss of a national resource whose education and training is largely federally supported. It is a cost we all eventually bear, one way or another. The actual cost to an organization of replacing a physician ranges from US $\$ 500,000$ to US $\$ 1,000,000$ and has been estimated to cost approximately US $\$ 2,000,000$ for a surgeon, specifically, in lost revenue, signing bonuses, start-up time, and salary. ${ }^{5}$

It is obvious that there are individual, organizational, and national imperatives for reversing the course of burnout and embracing what is known as the "Joy in Practice."

Addressing ways of preventing depression, depersonalization, and emotional exhaustion is a multidisciplinary effort involving administrators, organizational and clinical leadership, and surgeons themselves. There is no lack of interest in the problem of burnout and no controversy regarding its severity or detriment to the profession. However, a plethora of efforts by local administrators, health care chief executive officers (CEOs), national physician groups, and physicians themselves require organization, a certain amount of data-driven triage, and an ability to parse out the efforts that will provide the most relief most rapidly.

\section{Obstacles to Resilience}

A better understanding of challenges to resilience sets the stage for an exploration of the data regarding personal and organizational intervention. The five most common barriers to resilience include (1) an imbalance between work and personal life, (2) overexposure to stressful events, (3) insufficient time and space to process negative feelings, (4) humiliating experiences, and (5) social isolation. Mitigation of these circumstances forms the basis for both individual (personal) and structural (organizational) interventions to increase resilience (Dr. Anthony Rostain, personal communication). Dr. Ken Ginsburg and Dr. Anthony Rostain, clinicians at the University of Pennsylvania, who specifically study the role of individual and organizational attributes contributing to burnout in both adolescents and physicians, frame factors contributing to well-being as the "7 C's" of resilience: control, competence, confidence, contribution, coping, connection, and character (-Fig. 1). Effective interventions promoting resilience should ideally positively impact one or more of these factors.

\section{Surgeon Well-Being: Agency and the Individual}

In social science, "agency" describes the capacity of individuals to act independently and to make their own free decisions. In contrast, "structure" is those factors that determine or limit an agent or individual and his/her choices. In fact, psychologist Christina Maslach, an expert in the study of physician burnout, describes burnout as "not a problem of people, but of the social environment in which they work."7

The ability of any individual to effect change, participate in self-care, or even recognize factors that contribute to burnout or promote resiliency is partially dependent on certain personal factors and characteristics that may be unrelated to the clinical environment. The ability to have "agency" is at least partially predicated on the ability to recognize stressors, ability to mitigate them, and having the emotional intelligence necessary to build resilience and wellbeing.

Only three "individual" or personal interventions (those that can be practiced in the absence of an organizational mandate or structure) have been investigated in detail sufficient to assess efficacy in terms of meaningful, measurable, outcomes. Mindfulness, stress management, and small group discussions are thought to be at least partially effective in building resilience by specifically addressing the coping, character, and connection aspects of well-being.

"Mindfulness" is the psychological process of focusing on the present with the goal of developing wisdom through selfawareness by way of meditation, yoga, and other contemplative techniques. It has roots in Buddhist traditions and 


\section{C's Model of Resilience}

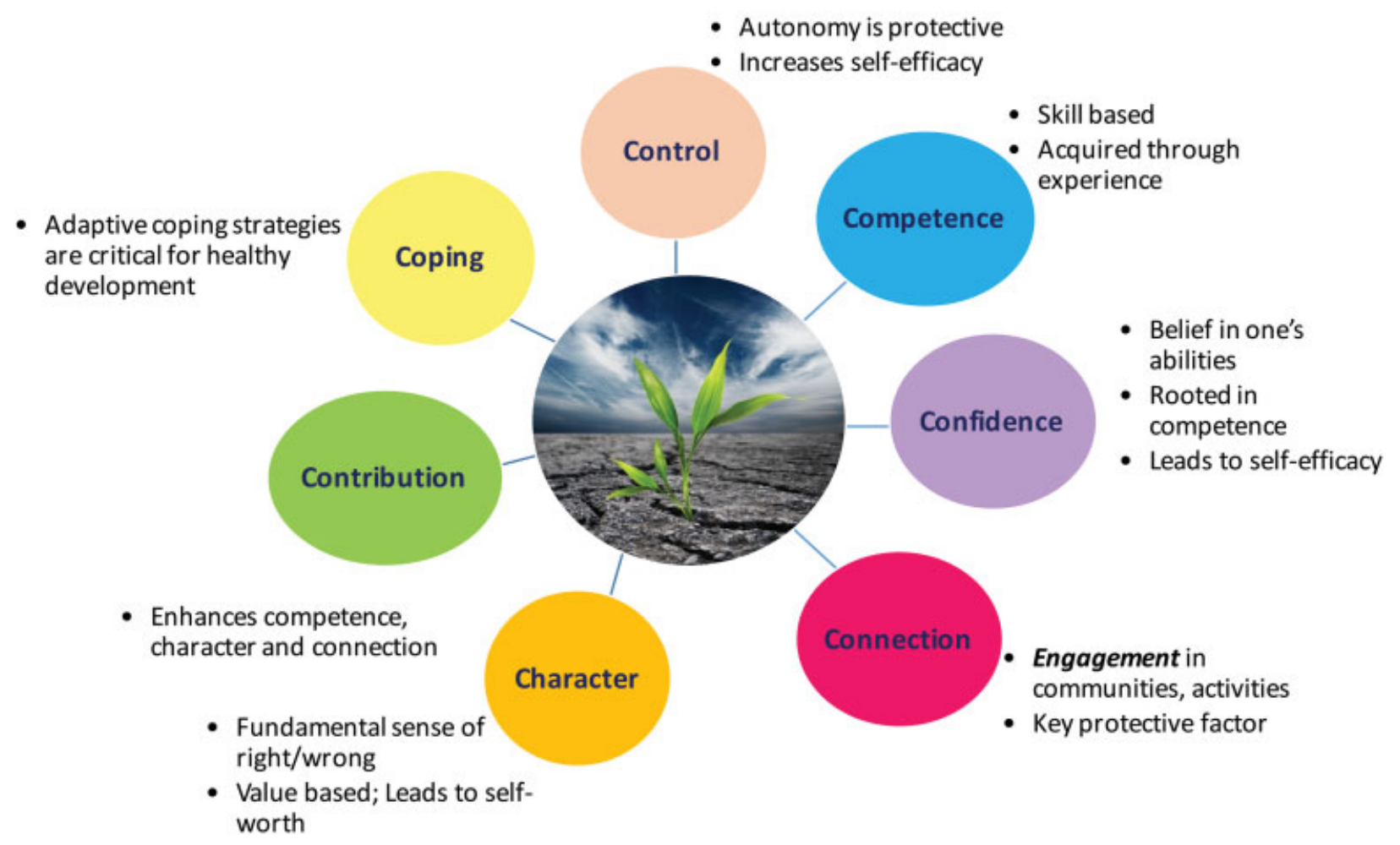

Fig. 1 The "7 C's" of resilience. (Reproduced with permission of Dr. Anthony Rostain.)

was described by Jon Kabat-Zinn at the University of Massachusetts in 1979 as a way to deal with persistent rumination, suffering, and worry in the chronically ill. ${ }^{8}$ It has lately been embraced by popular culture as well as a variety of institutions (schools, companies, hospitals) as a tool to help handle individual stress and emotional overload. It is a technique that can be practiced by individuals on their own terms-in their own space and on their own time, requiring no help, oversight, or guidance.

Stress management programs arose following the observation that many physicians have significant stressors both at work and at home that affect productivity and performance. Modern employee assistance programs have stress management programs that can be accessed by individuals independently and by referral. Individual intervention begins by assessing stressors by questionnaire or interview with a mental health professional followed by intervention aimed at changing behavioral patterns. Stress management programs can include counseling on stress management and administration of techniques such as relaxation, biofeedback, and cognitive restructuring. These programs can reduce the level of distress and help reframe a problem causing anxiety. Physicians who master behavioral and cognitive stress-relief techniques report less tension, fewer sleep disturbances, and improved coping strategies.

The Stress Management and Resilience Training (SMART) program was developed at the Benson-Henry Institute for Mind Body Medicine at Harvard. The concept was developed from an 8-week course model of stress reduction shown to help treat chronic diseases such as hypertension, chronic pain, depression, and anxiety. It had improved coping and decreased perceived stress among those in the armed forces and public school system, and it decreased stress and increased perspective-taking among palliative care clinicians at Massachusetts General Hospital. It has been studied only in resident physicians. The program emphasizes the use of mindfulness techniques to focus on priorities and alleviate stress. However, pilot programs and subsequent studies in this cohort failed to find significant benefit from this intervention after 6 months. It was found that burnout scores skyrocketed in interns from $39 \%$ baseline in June of internship year to $71 \%$ in January. ${ }^{9}$ Finding significant benefit from individualized solutions remains problematic. Study design is difficult, and reported outcomes as measured by composite burnout scores can be hard to interpret.

Dr. Tait Shanafelt (now Chief Wellness Officer for Stanford Medicine) and his group at Mayo Clinic have been pioneers in the study of physician burnout and interventions to promote resilience. His group examined the available literature in 2016 to see if the institution of policies to promote individual resilience (mindfulness, stress management techniques such as SMART, or group discussions) could make a difference in burnout scores. They found that overall burnout scores improved from $54 \%$ to $44 \%$ ( $p<0.001$ ) across the spectrum of the studies and across all interventions, but it was difficult to determine who was affected most and which interventions were most effective for which group. Importantly, it was impossible to determine whether reported scores were 
sustainable. ${ }^{10}$ Others have found that the sustainability of the effects of stress relief programs that relied on individually initiated participation was less than 6 months. ${ }^{11}$

Early intervention to provide individuals with tools to encourage resilience is felt to be very important (Dr. Juliette Galbraith, personal communication). Development of resilience begins at the start of surgical training and is promoted by developing a healthy "culture." Conversely, negative attitudes and the inability to cope are modeled with devastating efficacy by senior surgeons and adopted by our trainees. Ben Lovell describes how physicians, during medical school, find their own "group": clinicians with whom they identify and whose values and attitudes are shared. Surgical residents tend to socialize and feel comfortable in the company of other surgeons and either consciously or unwittingly integrate the knowledge, customs, values, and behaviors of those they admire and seek to emulate. ${ }^{12}$ Creation of a "professional identity" is a result and a direct consequence of the apprenticeship model that is at the heart of general surgery training. Clearly, this can be either good or bad-the consequences are largely created by the group environment and the actors within the group itself.

Self-identity and professional identity are tightly linked, especially in professions that require a large commitment of time and effort. But the process of linking these cognitive areas for trainees is dependent on ready access to the surgical community and the ability to participate. Membership in a professional community can be a very powerful bulwark against burnout and a tool to build resilience. Being part of a professional group can protect against burnout by engendering a sense of belonging and shared knowledge (both technical and emotional). It can foster the idea that the individual is a valued member of the society within the context of the group and is positively associated with the ability to handle stress.

In contrast, bad "modeling" is detrimental to the psychological health and well-being of the individual. Inability to find a suitable role model within a community of interest such as surgery is associated with depression and burnout and may explain, in part, why female surgeons and surgical trainees have higher rates of burnout. There are simply far fewer female role models within the larger community and therefore a higher rate of discordance between self and professional identity.

Cleland et al incorporated the social aspects of surgery into their "surgical boot camp" training. Surgical boot camp is a relatively new training method meant to acquaint interns in the first few days of training with the technical and medical tools that are important for them to know in their first year. Conceptually, familiarity reduces both stress and errors. Cleland et al decided to incorporate a community building facet into the boot camp, encouraging trainees to exchange stories, acquainting them with senior surgeons, and inviting them into their homes. Narrative data showed that the experience was powerfully positive for the trainees and resulted in a stronger, more positive sense of identification, belonging, and ability to share emotional experiences without fear of being judged harshly. ${ }^{13}$ The social elements of the boot camp were as important and affirming as the ones that focused strictly on medical knowledge. Therefore, it is important to be able to model appropriate behaviors and values ourselves, not just in the operating room or clinic but also emotionally and socially.

The concept of "self-complexity" is linked strongly to resilience and well-being. While a sense of "belonging" and community is associated with better well-being through shared experience, the ability to self-identify in a variety of ways-for example, gardener, father, mother, cyclist, traveler, music lover-is strongly correlated with emotional resilience. The occasional negative experience of one aspect of the self is balanced by the positive reinforcement created by success and community elsewhere in other arenas of experience. This is a very important concept to teach and activity to participate in. Finding a "hobby" or community of interest unrelated to surgery and nurturing it encourages self-complexity and is a proven defense against burnout. ${ }^{12}$

Discordance between the values of the institution and the individual is another major driver of burnout. As health care compensation becomes increasingly driven by factors that are not under the control of the physician, such as federally mandated quality initiatives, and billing becomes more complex, regulatory infrastructure can reach into the day-to-day lives of physicians and affect clinical decisionmaking in ways that impinge on autonomy and create a disconnect between what we think is important as physicians (direct patient care) and what is organizationally imperative (cost-effectiveness and avoidance of lost revenue). Finding meaningful clinical activities within our control that aligns with our own core values as physicians is shown to be an important personal strategy to avoid burnout. Devoting at least 20\% of the work week to these activities may foster well-being by directly aligning a sense of personal mission and agency with the expectations of our patients and our workplace. ${ }^{14}$

\section{Surgeon Well-Being: Organizational Responsibility and Action}

It is clear from the data on resilience that individual solutions to the problem are important, but supporting data are scant and difficult to interpret. It has been pointed out that even small improvements (as little as $1 \%$ in validated scores) can be measurably meaningful. Even so, institutional programs and mandates have an even larger impact on the development and maintenance of resilience and are better able to foster sustained change through organized large-scale solutions to issues that counter resilience. Christina Maslach and Tait Shanafelt have posited that the workplace is the most important factor in preventing burnout and fostering resilience-environment is the crucial element requiring change. ${ }^{7}$ Job engagement is the opposite of burnout, and identification of the structural causes of burnout within the organization, its "roots," is replacing older notions of blaming surgeons for poor coping skills. Also, implementation of solely individualized solutions may have unintended consequences-for example, reduction of one physician's 
workload may have the effect of increasing that of others. The role and responsibility of the organization in contributing to individual physician well-being is increasingly recognized and focused on specific programs and initiatives that positively impact workflow and allow for "perks" that make work-life balance more sustainable. The concept of "positive institutions" is defined as a place where the physician can develop, enrich, and sustain human potential. Institutional virtues in positive institutions include the following:

- Purpose: shared vision of moral goals.

- Safety: protection against threat, danger, and exploitation.

- Fairness: equitable rules governing reward and punishment, and the means of consistently enforcing them.

- Humanity: mutual care and concern.

- Dignity: treatment of all people as individuals regardless of their position.

Perhaps the most thorough explanation of the role of the organization to date originates from the Mayo Clinic, where Drs. Shanafelt (former Executive Director of Physician WellBeing), and John Noseworthy (CEO) have defined nine key characteristics of a healthy organizational structure promoting physician sustainability and resilience based on the extensive work done at Mayo Clinic on this subject in the last decade. They not only make a humanitarian argument for institutional consideration of physician well-being but also put forth a business case as well (-Table $\mathbf{1}$ ). The basic underpinnings of the proposal involve increasing engagement by identifying issues first and then directly addressing them at all levels including those in the highest leadership positions. Providing targeted interventions, correctly structuring incentives, providing resources to improve self-care, and aligning institutional values with those of physicians are key interventions that serve to increase control, competence, confidence, contribution, and connection-integral characteristics of Ginsburg's "7 C's" aspects of resilience that rely on structural support to flourish. ${ }^{15}$

Identification of issues at Mayo Clinic involves a variety of formats including small group discussion, "town hall" type meetings, and printed and video format messaging. Direct measurement of burnout scores is performed through the Mayo Well-Being Index, a validated instrument now used

Table 1 Organizational strategies promoting resilience

Organizational strategies to reduce burnout and promote physician engagement

Acknowledge and assess the problem

Harness the power of leadership

Develop and implement targeted work unit interventions

Cultivate community at work

Use rewards and incentives wisely

Align values and strengthen culture

Promote flexibility and work-life integration

Provide resources to promote resilience and self-care

Facilitate and fund organizational science

Source: adapted from Shanafelt and Noseworthy. ${ }^{15}$ (licensed) by other hospitals and health systems to collect self-reported data on metrics of resilience and burnout. Scores are compiled anonymously by division/department to learn where success or failure is most prevalent and to help direct resources. While not a perfect metric, this information gathering does give physicians a place to start instituting programmatic changes and a way to measure the efficacy of these alterations.

In this model, effective leadership is recognized as an evidence-based fortification against burnout. Data support the fact that small incremental increases in leadership skills result in a larger-than-expected decrease in burnout scores among faculty. Good leadership performance and its effects can be directly measured. Recognition of this fact mandates choice of good leaders based on known leadership skills-a merit-based leadership model rather than one rooted in academic or clinical performance or, worse yet, time served in the organization. Regular assessment of leadership by faculty, incentives based on leadership metrics, and scheduled performance reviews are all necessary for this aspect of prevention to thrive.

Specific drivers of burnout and modification of these factors are identified by comparing scores with external benchmarks to identify "high-opportunity" divisions where dissatisfaction and frustration are affecting performance and engagement. These factors are quite different between specialties, but common drivers include the electronic medical record or electronic health record (EHR) and the glut of electronic forms, "messaging boxes," billing and coding records, and extensive note formatting that they produce. It has been observed that the increase in burnout in the past decade directly correlates with the rise of the use of EHR. Targeting ways of streamlining EHR input, use of advanced practice providers to share in the burden, and employment of medical scribes and administrative assistants to off-load burdensome administrative and clerical tasks have all been effectively employed.

Organizational initiatives to promote flexibility and work-life integration can help with achieving balance. Almost half of all physicians work more than 60 hours per week, as opposed to less than $10 \%$ of the U.S. nonphysician workforce, and this is a considerable contributor to burnout. ${ }^{16,17}$ Providing flexibility in clinic or operating room hours that free up time elsewhere can help reconcile stressful disparities between the demands of family (still disproportionally problematic for women) and the needs of patients. Health system support staff flexibility (e.g., night or weekend hours) can be managed appropriately if needs are anticipated and planned for. Team workflow planning and engagement are necessary for a successful rollout of a flexible model of time management in an equitable manner.

Physicians have identified the concept of "loss of community" as a major issue that has evolved over time as unintended consequences of advancements in technology. Time spent doing clerical and administrative tasks, decentralization of offices, and repurposing physician's lounges all serve to erode direct interaction. The COMPASS (Colleagues Meeting to Promote and Sustain Satisfaction) groups started 
at Mayo Clinic involve specific dedicated time subsidized by the health system for colleagues to directly meet and discuss relevant issues related to culture, workflow, policy, and patient care. Dinners off-site are facilitated with the first 20 or 30 minutes spent examining issues related to the challenges and joys of being a physician. Participation in the COMPASS program (which is voluntary and therefore self-selected) resulted in an increase in engagement and work satisfaction. To date, about one-third of Mayo physicians have joined a COMPASS group.

The long-standing emphasis on relative value unit (RVU) based compensation may have contributed to the incidence of overwork, particularly for surgeons and proceduralists. Physicians have three major ways of increasing compensation: spend less time with each patient, order more tests, or work longer. Surgeons may be at a higher risk of overwork due to higher debt, longer training times, and training that can be militaristic with an emphasis on long work hours and endurance. Productivity-based pay using RVUs may serve to subtlety incentivize surgery for marginal indications, foster unhealthy competition for cases instead of collaboration, and encourage surgeons to overschedule. There is no standard pay model in the United States, but the most common are productivity only, base salary plus productivity bonuses, and (far less common) a pure salaried model. Adding other dimensions to compensation bonuses besides case volumes or RVUs are patient satisfaction scores and aspects of self-care and well-being. "Citizenship" in a division or department can be used to incentivize participation in quality programs with specific outcome metrics or in the teaching or academic mission of the institution. As mentioned earlier, it has been observed that physicians who allot $20 \%$ of their time to patient care pursuits they are passionate about are more resilient and express greater satisfaction at work. Building in a $20 \%$ incentive with metrics designed by the physician may be a way of marrying personal engagement with productivity. Many employees other than physicians and administrators are dependent on the financial success of our health systems, therefore careful planning and anticipation of downstream consequences of revenue changes must be predicted and accounted for. Even so, organizations that have instituted specific faculty engagement programs to address burnout and resilience, such as Mayo Clinic and Stanford University, have observed that the investment of resources to run programs is relatively low and the efficacy can be quite high, thus ensuring the long-term viability and financial feasibility of the institution as well as its employees. Mayo Clinic saw a 7\% reduction in burnout scores in the two years between 2011 and 2013, when national burnout scores rose by $11 \%$, after identifying and addressing the issues programmatically. ${ }^{17}$ It is likely that long-term investment consistently will produce the most durable results. Mayo Clinic has a burnout rate that is two-thirds of that of other organizations, but it is still $32 \%$. Although the pace of progress seems to be slow, cultural changes resulting in improvement in objective measurements of engagement are clearly occurring.

\section{Resilience and Burnout Prevention}

In 2013, a team of psychologists in Germany set out to determine the resilience strategies of experienced physicians by using objective instruments to measure coping skills and burnout and by eliciting narrative data through semistructured interviews. They found that measurements for both emotional exhaustion and depersonalization were similar between surgeons and other specialties and were detected in 26 to $32 \%$ of the respondents. Resilience strategies were grouped into three distinct categories: jobrelated gratification, personal routines to reinforce humanism, and positive attitude. Job-related sources of gratification involved enjoyment of the doctor-patient relationship and a sense that treatment was both appreciated and effective. Practices and routines contemplated building and maintaining relationships with both family and colleagues, preventing isolation both at home and at work; facing and dealing with complications directly and immediately; and setting aside time for personal reflection and goal setting. Attitudes of resilient physicians included acceptance and realism, self-awareness, recognizing when change is necessary, and active engagement with the downside of medicine. Getting involved in the organizational hierarchy to facilitate change and learning about sources of conflict and their underpinnings is a powerful way to facilitate both competency and control. ${ }^{18}$

Although primary prevention of burnout and promotion of resilience should be enacted through structural and cultural change at the organizational level where it is most effective, providing resources for the promotion of resilience remains an additional institutional responsibility. Tools for self-calibration like the Mayo Physician Wellbeing Index need to be linked to resources for the individual that help build resilience and reduce distress. Lowering the barriers to participation in exercise and fitness programs and providing ready-to-heat meals, breastfeeding rooms, facilitated medical appointments, and skills training in individual relaxation and stress reduction techniques can be helpful in the context of real organizational restructuring.

Data suggest that the best outcomes result from both individual and organizational interventions to build resilience. Those that are primarily individual or "personal" tend to have short-term results, whereas structural interventions are both longer lasting and more effective. Longitudinal investment in both types of activities is essential or else long-term gains will not be realized. ${ }^{11}$

\section{National Organizations' Responsibilities and Actions}

As noted by Shanafelt and Noseworthy, substantive progress is "unlikely to occur until there is a coordinated effort to address this issue at the national and state, organizational, leader, and individual levels." ${ }^{15}$ In the absence of such a coordinated effort, the epidemic of burnout continues to worsen. 
Today, many physicians and health care leaders agree with the sentiment recently expressed by Dzau et al that "the health professions are at a critical inflection point. ${ }^{5}$ The health system cannot sustain current rates of clinician burnout and continue to deliver safe, high-quality care." This sense of urgency has prompted several national health care organizations to develop responses to the burnout crisis. Their efforts are focused primarily on the following two broad areas: (1) restoration of clinician well-being and (2) implementation of needed health care reforms.

National Academy of Medicine (NAM): a major barrier to reversing the current trend of physician burnout is the absence of a national clearinghouse to systematically gather and compare data in order to scientifically determine what works and what does not. To overcome this deficiency, the NAM (formerly the Institute of Medicine or IOM) recently accepted the responsibility "to coordinate and synthesize the many ongoing efforts within the health care community and to generate momentum and collective action to accelerate progress." ${ }^{5}$ Additionally, as part of its coordinating role, NAM also committed to involve "key influencers beyond the health care community such as information technology (IT) vendors, payers, regulators, accreditation agencies, policymakers, and patients." 5

Action Collaborative on Clinician Well-Being and Resilience: In January 2017, in collaboration with the Association of American Medical Colleges (AAMC) and the Accreditation Council for Graduate Medical Education (ACGME), NAM launched a national "Action Collaborative on Clinician Well-Being and Resilience." The Action Collaborative's initial goals ( - Table 2 ) focused on increasing awareness and understanding of physician burnout and identifying and implementing evidence-based solutions at the organizational, system, and cultural levels. Initially, at least, the Action Collaborative chose not to focus on personal factors unrelated to the clinical environment. By January 2018, 55 core organizations and a network of more than 80 others (including specialty and other professional organizations), as well as payers, researchers, government agencies, technology companies, patient organizations, and trainees, had joined the Action Collaborative. Four workgroups are now charting their initiatives (-Table 3 ). The Action Collaborative also has pledged to launch an online "knowledge hub" in 2018. The hub is envisioned as a user-friendly repository for available data, models, and toolkits and as a site for clinicians and other stakeholders to share information and build productive relationships.

Table 2 Initial goals of the action collaborative

\begin{tabular}{|l|}
\hline Increase visibility of physician stress and burnout \\
\hline $\begin{array}{l}\text { Improve health care organizations' baseline understanding } \\
\text { of the challenges to clinician well-being }\end{array}$ \\
\hline Identify evidence-based solutions \\
\hline $\begin{array}{l}\text { Monitor effectiveness of implementation of } \\
\text { these solutions }\end{array}$ \\
\hline
\end{tabular}

Source: based on information from Dzau et al, page $313 .^{5}$
Table 3 Workgroups of the action collaborative

\begin{tabular}{|c|}
\hline Research, data, and metrics \\
\hline $\begin{array}{l}\text { Compiling validated survey instruments } \\
\text { Compiling validated evidence based interventions } \\
\text { Identifying benchmarks for gauging progress in } \\
\text { supporting clinician well-being }\end{array}$ \\
\hline Conceptual model \\
\hline $\begin{array}{l}\text { Created a comprehensive conceptual model } \\
\text { Establishing shared taxonomy by defining key factors }\end{array}$ \\
\hline $\begin{array}{l}\text { External factors and work flow: assessing approaches to } \\
\text { achieve optimal }\end{array}$ \\
\hline $\begin{array}{l}\text { Team-based care } \\
\text { Documentation in rapidly evolving digital health } \\
\text { environment }\end{array}$ \\
\hline Messaging and communications \\
\hline $\begin{array}{l}\text { Identifying key stakeholders } \\
\text { Developing targeted messaging to disseminate } \\
\text { available evidence and knowledge } \\
\text { Inspiring action at local level }\end{array}$ \\
\hline
\end{tabular}

Source: based on information from Dzau et al, page $313 .^{5}$

ACGME Common Program Requirements: in recognition of the importance of physician burnout, Section VI of the ACGME Common Program Requirements (effective July 1, 2017) has been amended to include a "commitment to the well-being of the students, residents, faculty members, and all members of the health care team." That new commitment is detailed in a new "Section VI.C. Well-Being," which begins by acknowledging the gravity and scope of the problem: "In the current health care environment, residents and faculty members are at increased risk for burnout and depression. Psychological, emotional, and physical well-being are critical in the development of the competent, caring, and resilient physician. Self-care is an important component of professionalism; it is also a skill that must be learned and nurtured in the context of other aspects of residency training. Programs, in partnership with their sponsoring institutions, have the same responsibility to address well-being as they do to evaluate other aspects of resident competence."19

In addition to that new section on physician well-being, Section VI has also been extensively revised to focus on "The Learning and Working Environment." The newly revised Section VI is a useful template for nonteaching hospitals and health care institutions to adopt, as they strive to mitigate burnout and promote well-being by improving their workplace environment. In particular, portions of Section VI address patient safety, quality improvement and accountability, professionalism, fatigue mitigation, and teamwork and transitions of care.

\section{Increasing Clerical Burdens and the Cost of Good Intentions}

Since 2000, high-technology health care work environments have become commonplace across the United States. Their features include the mandated use of EHRs, computerized 
physician order entry and billing, patient portals, and around-the-clock remote computer access to patient records, test results, and inpatient status. Paradoxically, these technologies were justified as indisputable ways to improve patient care and lighten the workload of physicians and other health care providers, but today they are a major source of physician dissatisfaction and burnout. Dr. Christine Sinsky, a practicing internist and vice president for professional satisfaction at the American Medical Association (AMA), estimates that the modern health care workplace is likely causing $80 \%$ of the burnout among American physicians (Christine Sinsky, M.D., personal communication, September 22, 2016). ${ }^{14}$

In a recent Perspective article published in the New England Journal of Medicine, Wright and Katz noted that the increasing clerical burden is one of the biggest drivers of burnout and that such work is generally not reimbursed but rather is done "in the interstices of life, during time often referred to as 'work after work'-at night, on weekends, even on vacation." ${ }^{20}$ Wright and Katz cite the analysis of such "work after work" done by Dr. Shanafelt. He argues that such a practice "has enabled organizations to continuously increase productivity targets without changing the infrastructure or support system, effectively adding a whole extra work week hidden within a month." Sinsky agrees, noting that "this assumption that doctors could take on extra work seamlessly, but now it is crowding out our true work as healers. Physicians are at the sharp end of the stick for accountability, regulatory issues, and now even data acquisition and entry - it's too much."20

Accompanying this increasing clerical burden is widespread cynicism about why it is required in the first place. Increasingly, many physicians and surgeons suspect that doctors are required to endlessly document all they do to justify the high costs and billing practices that are now part of modern health care. Worse, many doctors acknowledge privately that they are "encouraged" to perform and document unnecessary elements of care to justify billing codes.

Shanafelt et al have called for specific reforms at the national and state levels in multiple $\operatorname{areas}^{21}$ (-Table 4). Collectively, these reforms will begin to restore time for physicians and surgeons to concentrate on what we were trained to do, namely to be doctors. Our primary professional obligations are to understand and meet the needs of each of our patients and to be their advocate in today's complex, modern health care system.

\section{Summary and Conclusion}

Creating a respectful, effective, efficient, and sustainable workplace environment that fosters clinician well-being-while simultaneously making it easier to provide safe, high-quality care and appropriate services at a reasonable cost to all people living in our country-is a worthy but elusive goal. As described earlier, many physicians and surgeons across the United States have independently developed personal routines and methods to protect themselves from burnout and its sequelae; many local health care organizations have launched multiple, wellintentioned initiatives and programs to eliminate or mitigate burnout and restore well-being in their environment. Some of those efforts have been at least partly effective, but others have failed. The epidemic of burnout has worsened.

The rapid rise in the prevalence of burnout is, in part, a consequence of the unintended net result of multiple highly disruptive changes occurring in recent years. No individual physician/surgeon and no local organization can address all the societal, financial, political, legal, technological, scientific, and professional issues that contribute to burnout. It is encouraging that NAM, AAMC, and ACGME are collaborating to prioritize well-being, but they and other national organizations must also assume the responsibility to promote other needed reforms.

Table 4 Recommended national and state reforms

Reduce and streamline required documentation in the medical record and determine what elements must be completed by physicians and what elements can be completed by advanced practice providers and/or other appropriately trained nonphysicians

Seek broad input from physicians practicing in diverse settings and specialties to improve current and future regulations Improve the preapproval process for tests, medications, and procedures

Integrate maintenance of certification requirements with standard continuing medical education requirements

Eliminate questions on licensing applications related to diagnosis or treatment for mental health conditions and replace them with questions related to current impairment

Advocate that the National Institutes of Health increase funding of research to evaluate implications of clinician well-being for the care delivery system and determine how to improve the work and life of health care professionals

Encourage health care institutions to

routinely assess, monitor, and improve dimensions of well-being as a standard component of their performance metrics develop new practice models that increase efficiency and productivity while lessening the dependence on physicians performing clerical and administrative tasks

acknowledge the high work hours (including many uncompensated hours of "work after work") for physicians and work to improve flexibility in their schedules and enhance work-life integration prioritize physician well-being

Source: based on information from Shanafelt et al. ${ }^{21}$ 
In the meantime, local organizations do have the obligation to keep striving to (1) understand the factors underlying burnout in their own environment and (2) find ways to change their own culture to better support well-being. And, as individuals, surgeons and other physicians have a personal and professional responsibility to keep striving to take care of ourselves and each other, beginning with accepting the limits of our species. We are not superhuman-we need adequate sleep, good nutrition, and time to meet our own medical needs. We need positive working relationships with our colleagues and with our patients. We need time for our loved ones and our friends. We need private time to think, enhance self-awareness, restore energy and resiliency, and nurture both our personal and professional priorities.

\section{Conflict of Interest}

None.

\section{References}

1 Bosk C. Forgive and Remember: Managing Medical Failure, 2nd ed. Chicago, IL: University of Chicago Press; 2003

2 Shanafelt TD, West CP, Sloan JA, et al. Career fit and burnout among academic faculty. Arch Intern Med 2009;169(10):990-995

3 Andrew LB. Physician Suicide. https://emedicine.medscape.com/ article/806779-overview. Accessed June 12, 2017

4 Brandt ML. Sustaining a career in surgery. Am J Surg 2017;214 (04):707-714

5 Dzau VJ, Kirch DG, Nasca TJ. To care is human - collectively confronting the clinician-burnout crisis. N Engl J Med 2018;378 (04):312-314

6 Swensen SJ, Shanafelt T. An organizational framework to reduce professional burnout and bring back joy in practice. Jt Comm J Qual Patient Saf 2017;43(06):308-313

7 Maslach C, Leiter MP. New insights into burnout and health care: Strategies for improving civility and alleviating burnout. Med Teach 2017;39(02):160-163
8 Ludwig DS, Kabat-Zinn J. Mindfulness in medicine. JAMA 2008; 300(11):1350-1352

9 Chaukos D, Chad-Friedman E, Mehta DH, et al. SMART-R: a prospective cohort study of a resilience curriculum for residents by residents. Acad Psychiatry 2018;42(01):78-83

10 West CP, Dyrbye LN, Erwin PJ, Shanafelt TD. Interventions to prevent and reduce physician burnout: a systematic review and meta-analysis. Lancet 2016;388(10057):2272-2281

11 Awa WL, Plaumann M, Walter U. Burnout prevention: a review of intervention programs. Patient Educ Couns 2010;78(02):184-190

12 Lovell B. How to build a resilient surgeon: communities, cultures and complexity. Med Educ 2016;50(08):800-802

13 Cleland J, Walker KG, Gale M, Nicol LG. Simulation-based education: understanding the socio-cultural complexity of a surgical training 'boot camp'. Med Educ 2016;50(08):829-841

14 Rothenberger DA. Physician burnout and well-being: a systematic review and framework for action. Dis Colon Rectum 2017;60(06): 567-576

15 Shanafelt TD, Noseworthy JH. Executive leadership and physician well-being: nine organizational strategies to promote engagement and reduce burnout. Mayo Clin Proc 2017;92(01):129-146

16 Shanafelt TD, Boone S, Tan L, et al. Burnout and satisfaction with work-life balance among US physicians relative to the general US population. Arch Intern Med 2012;172(18):1377-1385

17 Shanafelt TD, Hasan O, Dyrbye LN, et al. Changes in burnout and satisfaction with work-life balance in physicians and the general US working population between 2011 and 2014. Mayo Clin Proc 2015;90(12):1600-1613

18 Zwack J, Schweitzer J. If every fifth physician is affected by burnout, what about the other four? Resilience strategies of experienced physicians. Acad Med 2013;88(03):382-389

19 ACGME. Common Program Requirements. Section VI with Background and Intent. https://www.acgme.org/Portals/0/PFAssets/ ProgramRequirements/CPRs_Section\%20VI_with-Background-and -Intent_2017-01.pdf. Accessed March 2, 2018

20 Wright AA, Katz IT. Beyond burnout - redesigning care to restore meaning and sanity for physicians. N Engl J Med 2018;378(04): 309-311

21 Shanafelt TD, Dyrbye LN, West CP. Addressing physician burnout: the way forward. JAMA 2017;317(09):901-902 